\title{
Prehospital evaluation and economic analysis of different coronary syndrome treatment strategies - PREDICT - Rationale, Development and Implementation
}

Laurie J Morrison ${ }^{1,2^{*}}$, Valeria E Rac ${ }^{1,3}$, James M Bowen ${ }^{4}$, Brian Schwartz ${ }^{5}$, Tyrone Perreira ${ }^{1}$, Welson Ryan ${ }^{1}$, Cathy Zahn', Rishab Chadha', Alan Craig ${ }^{1,6}$, Daria O'Reilly ${ }^{4}$ and Ron Goeree ${ }^{4}$

\begin{abstract}
Background: A standard of prehospital care for patients presenting with ST-segment elevation myocardial infarction (STEMI) includes prehospital 12-lead and advance Emergency Department notification or prehospital bypass to percutaneous coronary intervention centres. Implementation of either care strategies is variable across communities and neither may exist in some communities. The main objective is to compare prehospital care strategies for time to treatment and survival outcomes as well as cost effectiveness.
\end{abstract}

Methods/Design: PREDICT is a multicentre, prospective population-based cohort study of all chest pain patients 18 years or older presenting within 30 mins to 6 hours of symptom onset and treated with nitroglycerin, transported by paramedics in a number of different urban and rural regions in Ontario. The primary objective of this study is to compare the proportion of study subjects who receive reperfusion within the target door-toreperfusion times in subjects obtained after four prehospital strategies: 12-lead ECG and advance emergency department (ED) notification or 3-lead ECG monitoring and alert to dispatch prior to hospital arrival; either with or without the opportunity to bypass to a $\mathrm{PCl}$ centre.

Discussion: We anticipate four challenges to successful study implementation and have developed strategies for each: 1) diversity in the interpretation of the ethical and privacy issues across 47 research ethics boards/ commiittees covering 71 hospitals, 2) remote oversight of data guardian abstraction, 3) timeliness of implementation, and 4) potential interference in the study by concurrent technological advances. Research ethics approvals from academic centres were obtained initially and submitted to non academic centre applications. Data guardians were trained by a single investigator and data entry is informed by a detailed data dictionary including variable definitions and abstraction instrucations and subjected to error and logic checks. Quality oversight provided by a single investigator. The window of the trial in each community has been confirmed with the basehospital medical director to correspond to the planned technological advances of the system of care. We hope this comparative analysis across treatment strategies for clinical outcomes and cost will provide sufficient evidence to implement the superior strategy across all communities and improve outcomes for all STEMI patients.

Trial registration: ClinicalTrials.gov: NCT00747656

\footnotetext{
* Correspondence: MorrisonL@smh.ca

'Rescue, Keenan Research Centre, Li Ka Shing Knowledge Institute, St.

Michael's Hospital, University of Toronto, Toronto, Ontario, Canada

Full list of author information is available at the end of the article
} 


\section{Background}

Cardiovascular disease accounts for more deaths than any other disease and ischemic heart diseases, such as acute myocardial infarction (AMI), account for a large proportion of these deaths [1-3]. Timely recognition and reperfusion are life saving interventions [3-7]. Randomized controlled trials have demonstrated the superiority of prehospital fibrinolysis [8-11]; whereas other interventions such as 12-lead [12-15] and bypass to interventional hospitals [6,16-23] have not been subjected to the same rigorous analysis. Based on associations with improved time to treatment, 12-lead ECG and bypass to an interventional hospital has been implemented in many communities which makes it difficult to conduct a controlled trial. In contrast, many rural and urban communities with small volumes may not have implemented any one of these interventions, as such the standard of care for patients presenting with ST-segment elevation myocardial infarction (STEMI) varies between communities [24]. In 2004, the expert panel of Cardiac Care Network of Ontario report recommended that urgent angioplasty should be adopted as the standard of care[24]. Concurrent with this report the Ontario Health Technology Advisory Committee (OHTAC), upon their review of the literature regarding primary angioplasty for the treatment of STEMI, recommended that "every effort should be made to decrease the access time for patients with AMI from onset to symptoms to administration of fibrinolysis or primary angioplasty" $[25,26]$. This study is being conducted to evaluate different implementation strategies in place currently that could reduce symptom to intervention time in Ontario[25]. The objective of this paper is to describe the design of a study to compare these strategies against time to treatment and survival outcomes as well as cost effectiveness. We hope that this prospective cohort trial will promote the adoption of the optimal implementation strategy into the healthcare system and may provide the information required to directly change health policy and funding for systematic multidisciplinary care involving local EMS systems. We anticipate that the identification and implementation of the best care strategy may provide consistent and optimal care of patients presenting with STEMI across all communities in Ontario,

\section{Methods/Design Study Design}

PREDICT is a prospective, population-based cohort study of four patient care strategies provided by regional EMS services to patients with chest pain and suspected ischemia.

1. 3-lead PHECG and transported to the nearest receiving $\mathrm{ED}$ who were not eligible for bypass based on transport time.
2. 3-lead PHECG and transported to the nearest receiving $\mathrm{ED}$ who were eligible for bypass based on transport time, if 12 lead PHECG was possible.

3. 12-lead PHECG and prehospital notification transported to the nearest receiving ED who were not eligible for bypass to a PCI center based on transport time.

4. 12-lead PHECG with prehospital notification and eligible for bypassing the nearest receiving ED with transport to a PCI center.

Bypass eligibility was based on transport distance of patients from their pick-up location to PCI center and the cut-off point was 60 kilometres.

\section{Inclusion and Exclusion Criteria \\ Inclusion criteria}

$\circ$ Patients who call 911, and are:

- Suspected by the paramedics to have ischemic chest pain for greater than 30 minutes but less than 6 hours, and

$\circ 18$ years of age or older

$\circ$ Experiencing chest pain that fails to resolve with nitrates given as per protocol

\section{Exclusion Criteria}

$\circ$ Age $<18$ years of age

\section{Setting}

This study is set in regions of Ontario with a population of $3,043,853$ served by 14 EMS services, under the medical control of 4 regional Base Hospital programs (Table 1) [27]. These regions represent $25 \%$ of the population of Ontario and $9.6 \%$ of the population of Canada. This geographic region covers $206,727 \mathrm{~km}^{2}$ with variable

Table 1 List of regional base hospital programs and emergency medical services participating in PREDICT study

\begin{tabular}{ll}
\hline $\begin{array}{l}\text { Regional Basehospital } \\
\text { Programs }\end{array}$ & $\begin{array}{l}\text { Participating Emergency Medical } \\
\text { Services (EMS) }\end{array}$ \\
\hline $\begin{array}{l}\text { Hamilton Health Sciences } \\
\text { Centre for Paramedic }\end{array}$ & $\begin{array}{l}\text { County of Brant Ambulance Service } \\
\text { Haldimand EMS } \\
\text { Education \& Research - } \\
\text { Hamilton }\end{array}$ \\
$\begin{array}{ll}\text { Hamilton EMS } \\
\text { Hamilton-Wentworth Regional } \\
\text { Ambulance Service } \\
\text { Norfolk EMS }\end{array}$ \\
\hline Sorth-East Ontario Regional & Algoma EMS \\
Base Hospital Program - & Manitoulin-Sudbury EMS \\
Sudbury & Sudbury EMS \\
& Sault Ste. Marie EMS \\
\hline Northwest Region Base Hospital & Superior North EMS \\
Program & \\
\hline Sunnybrook Osler Centre for & County of Simcoe Paramedic Services, \\
Prehospital Care - Toronto & Peel Regional Paramedic Services \\
\hline
\end{tabular}


population densities from 0.6 to 574 persons per $\mathrm{km}^{2}$ representing rural, suburban, urban, and metropolis areas [27].

\section{Sample Size Calculation}

The recruitment goal is to enrol 100 STEMI prospective subjects per group (e.g. as in the WEST trial)[28], for a total of 400 STEMI subjects. The primary estimate is based on a difference in the proportion of patients who received reperfusion (fibrinolysis or PCI) within target door-to-intervention times. We based our calculation using estimates from Canto et al., 2002[29]. In that study there was an increase in the percentage of the patients who received lytic therapy within 30 minutes, from $31 \%$ to $50 \%$ (an absolute difference of $19 \%$ ). The percentage of patients who received PCI within $90 \mathrm{~min}-$ utes increased from $29 \%$ to $48 \%$ (an absolute difference of $19 \%$ ). Furthermore there was a combined $24 \%$ higher odds of receiving fibrinolytic therapy or PCI with the active EMS involvement (odds ratio 1.24, 95\% CI 1.21 to $1.28, \mathrm{p}<0.001)$. Calculations were conducted using PASS software, assuming an alpha $(\alpha)$ of 0.05 and power of $80 \%$ (Table 2).

To estimate the number of potential subjects that could be enrolled in the study, the annual rate of STEMIs that would occur within a 60 minute transport time of the closest PCI centre was determined. The surrounding areas within 60 minutes of a PCI centre were first identified using data from a Cardiac Care Network of Ontario (CCN) report published in 2004[6]. Current population estimates were then assigned to each of the surrounding areas using population estimates for 2006 [27]. For counties or regions where a proportion of the population resided outside a 60 minute radius, population data from the 2006 Canadian census was used from the census subdivisions to adjust the 2006 population estimates[27]. To determine the rate of STEMI, an estimate of 571 per 1,000,000 inhabitants was calculated by taking an estimate obtained using CIHI data of 6524 STEMIs in Ontario for fiscal 2001/02 and dividing it by the 2006 Ontario Census Population and determining the rate per million inhabitants[6,27]. The number of potential subjects to be entered in per year was then estimated by assuming a $50 \%$ transport by EMS rate and a potential recruitment rate of $70 \%$.

Table 2 Sample size calculation for the PREDICT study

\begin{tabular}{ccccc}
\hline Baseline \% & Intervention \% & $\begin{array}{c}\text { Absolute } \\
\text { Difference }\end{array}$ & Odds Ratio & $\begin{array}{c}\text { Number per } \\
\text { arm }\end{array}$ \\
\hline 37.4 & 47.4 & $10 \%$ & 1.5 & 402 \\
\hline 37.4 & 52.4 & $15 \%$ & 1.8 & 185 \\
\hline 37.4 & 57.4 & $20 \%$ & 2.3 & 106 \\
\hline
\end{tabular}

\section{Study Outcomes}

Primary Outcome

The primary outcome of this study is to compare the proportion of study subjects who receive reperfusion within the target door-to-reperfusion times across the four care strategies. Target door to reperfusion times are 90 minutes for primary PCI intervention (door-toballoon time) and 30 minutes for fibrinolysis (door-toneedle time)[30-32].

\section{Secondary Outcomes \\ Survival}

Survival at 30 days and one year after episode date (brief telephone assessment) for STEMI patients

\section{Treatment Time Intervals}

- Prehospital scene time interval defined as time from arrival at scene to departure from scene;

- Transport time interval defined as time from departure from scene to arrival at destination hospital;

- Symptom onset time interval defined as time from symptom onset reported by subject to reperfusion intervention (defined as time to drug administration or balloon inflation);

- Primary hospital reperfusion time interval defined as the time from arrival at primary destination hospital to reperfusion intervention at the primary destination (defined as time to drug administration or balloon inflation);

- PCI transfer reperfusion time interval defined as the time from arrival at primary destination hospital and transport to a PCI capable site to the reperfusion intervention at the PCI site (defined as time to drug administration or balloon inflation).

- PCI site reperfusion time interval defined as the time from arrival at PCI site to reperfusion intervention at the PCI site (defined as time to drug administration or balloon inflation)

\section{STEMI Identification with 12-Lead PHECG}

- Proportion of STEMI subjects within the target doorto-reperfusion times comparing basic vs. advanced life support paramedics.

- Paramedics and computer software interpretation of the 3-lead and 12-lead PHECG of all STEMI subjects will be compared with a gold standard (defined as consensus between two investigators' independent interpretation blinded to paramedic or software interpretation)

\section{Access to Interventions}

The rate of reperfusion strategy utilization across groups including fibrinolysis, percutaneous coronary angiography and intervention, coronary artery bypass surgery, bypass to PCI centre directly vs. transfer from a non PCI centre. 


\section{Economic Outcomes}

- The direct costs of the 12 lead PHECG program will be estimated.

- Impact on life expectancy gains through reductions in mortality based on the age and gender of subjects [33-35];

- Cost savings with survival benefits for the dominant treatment strategy or cost and outcome tradeoffs if one treatment strategy demonstrates cost increasing with survival benefits

- Incremental cost-effectiveness, as measured through additional cost per reduction in door-toreperfusion time and additional cost per life year gained, will be calculated [36,37].

\section{Patients Enrolment}

A single trained data guardian/abstractor at each base hospital will screen all ambulance call reports and identify all eligible cases. Trained inhospital data abstractors will be notified to conduct a chart review at each receiving hospital.

\section{Data Management}

All prehospital and inhospital data will be abstracted by trained staff and entered on a web based interface employing a structured data set (Additional file 1 Prehospital Data Variables and Additional file 2 - Inhospital Data Variables) that complies with institutional, privacy and ethical requirements. A manual of operations defines the data name, definition and abstraction instruction for each variable. Error and logic checks were built into the database to screen for abnormal values across forms and within forms at point of entry.

\section{Analysis Plan}

The primary outcome, and treatment time intervals, will be analyzed with one-way ANOVA and subsequent pair-wise multiple comparison procedures across the four treatment strategies. Different covariates (Table 3) will be analyzed using multiple linear regressions and they will be introduced into the model and evaluated as potential confounders. Variables will be retained if they have had an effect of $5 \%$ or greater on the coefficients for door-to-reperfusion time. Survival at 30 days and one year for STEMI patients will be analyzed as a binary outcome (Chi square) and as a survival analysis. Covariates that may affect survival will be analyzed with a Cox PH Regression Model. Adverse event rates will be analyzed with a Chi square analysis or a Fisher's exact test.

Economic outcomes will be analyzed using decision analysis supplemented with probabilistic sensitivity analysis in a simulation model. Direct costs will be estimated for each of the four treatment strategies.

\section{Table 3 Analysis Plan - List of Covariates}

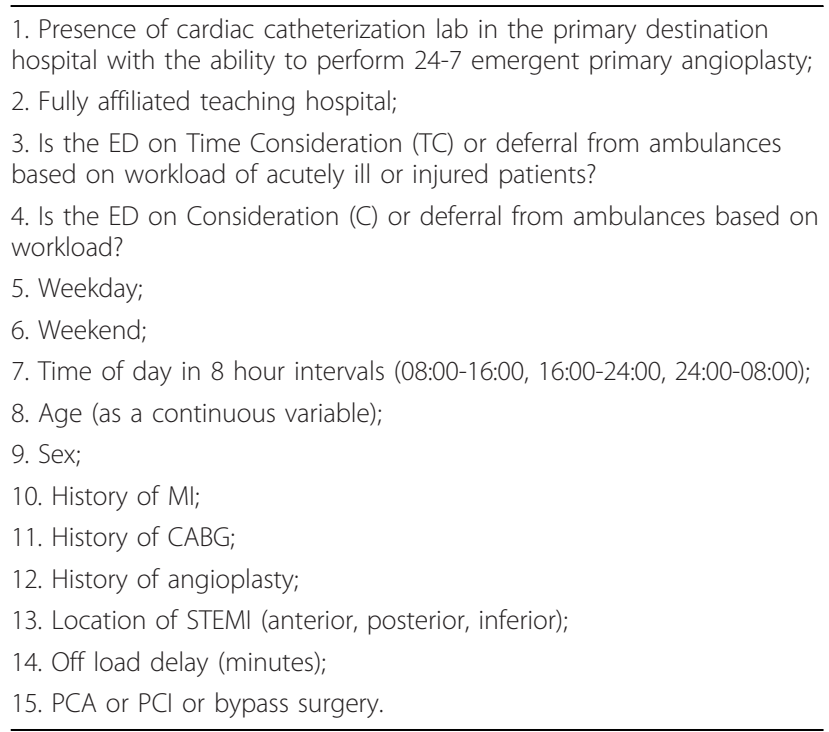

Door-to-reperfusion times and mortality will be available at the patient level, which will allow for the calculation of averages as well as variability estimates for analysis of uncertainty. Average cost and effectiveness (time-to-reperfusion interval and life years) will be calculated and if one treatment strategy is found to be superior (i.e. cost savings with survival benefits), and then these results will be reported in a cost consequence format. If the superior strategy is found to involve cost and outcome trade-offs (i.e. cost increasing with survival benefits), then incremental cost-effectiveness, as measured through additional cost per reduction in time-toreperfusion interval and additional cost per life year gained, will be calculated.

\section{A Priori Subgroup Analysis}

- Rural vs. urban settings and academic vs non academic destination hospitals

- Geographical bias subgroup analysis comparing all non PCI capable sites for distance from PCI site;

\section{Ethical Considerations and Human Subjects Protection}

PREDICT is an observational, prospective non-interventional study based on review of routinely collected source data and as such meets the requirements for minimal risk research[38-40]. Approval by 47 research ethics boards/committees covering 71 hospitals will be sought to launch the study.

\section{Discussion}

There is a lack of a comprehensive dataset for Acute Coronary Syndrome (ACS) patients, which includes the prehospital component of care[3]. We anticipate that 
this study will bridge this gap, providing valuable information on processes of care and the benefits of different prehospital treatment strategies. We have planned to address four threats to protocol compliance and internal validity; 1) ethics approval and privacy requirements from 47 research ethics boards/committees covering 71 hospitals, 2) temporal bias of comparison induced by delays to implementation across sites, 3) data guardian training and oversight of timeliness and quality, and 4) technological advances that may outpace the study and affect recruitment.

This trial involves rural and urban centres and this means that many research ethics boards will need to review this protocol and our request for waiver of consent. We anticipate that rural and small community hospitals will struggle with the request for waiver of consent and the privacy issues associated with chart abstraction, acquisition of personal information enabling telephone follow up at 30 days and at one year. Our strategy will be to obtain approval from all the academic centres first and enclose a copy of their approval with submission to the smaller centres. In addition we have established a data sharing agreement template that has the approval of the administration and legal advisors of the 18 academic and community hospitals in our largest metropolitan area. This agreement has been used successfully in other trials and we hope this will facilitate reaching agreement more quickly with hospitals that have not been involved with research previously. And, finally our investigators will be on call to the research ethics board to participate in the discussion at the time of review. Many small hospital boards request this level of participation by the investigator. We have found in the past that this strategy is helpful in minimizing correspondence back and forth between the research team and the ethics board and reduces time to approval.

We recognize the limitations and challenges that might affect the study's successful implementation and generalizability such as time and spatial challenges. If at all possible, the study should be launched in all centres at the same time. However prior to launching, each PREDICT participating site will need to confer with stakeholders to ensure capture of all cases, procurement of all source documents, optimization of timely data flow, and training of data abstractors. For some centres this preparation and initiation phase will be more elaborative and time consuming then for the others based on volume and existing infrastructure and prior research experience. To address these potential delays we will target the sites we anticipate the REB will be slower to approve the protocol, with follow up calls and offers to complete additional information or speak by teleconference to the board or to the ethics board chair to facilitate understanding and a timely response to queries.
We will engage the medical directors in all the sites to ensure the flow of documentation allows for timely data entry and to encourage them to identify and support a high quality data guardian for their site. This engagement will take the form of web based reports of site performance and patient outcomes available 24-7 that enable medical directors to see their data and compare to the aggregate site data. To date most medical directors do not have access to performance or outcome data and we hope providing this accessibility will speed implementation and timely quality data. We anticipate these interventions may be sufficient to allow all sites to participate concurrently and limit the bias related to temporal changes in practices during the trial.

By protocol, the data is collected by trained data guardians in all the sites and since almost all the data guardians are geographically remote from the central research coordinating centre the quality of data may suffer and poise a threat to internal validity. In total there are 10 data guardians abstracting prehospital data and 16 data guardians abstracting inhospital data. To encourage uniformity in data collection and to provide oversight and ensure quality, a number of interventions are planned. All data guardians will be individually trained by one of the investigators (VR). The web based data entry system has built in data definitions and abstraction instructions which are accessible through point and click technology on the variable name at the time of data entry. The abstraction instructions are listed hierarchically ensuring that the data is abstracted from the best source if at all possible. All variables are subject to error and logic checks across other variables and across forms (inhospital and prehospital) which are applied at the time of completion and the case will not close without reconciliation of all the error. Web conferences are conducted for all data guardians to highlight changes to the data set structure, upgrades to the software and discuss difficult variables identified by the data guardians or by the investigators. Data reports to test uniformity are planned and will be discussed at weekly team meetings of the research staff and investigator steering meetings.

Technological advances may outpace the study. Some regions/counties that provide 3-lead ECG in the prehospital setting are not currently considering the change in technology, while other areas are in the planning or transitional stages. Any change from 3-lead to 12-lead in a participating site will compromise recruitment rates and regional comparisons. If this happens an additional 3-lead site with similar geographic and demographic characteristics will be recruited and retrospective data collection will occur to permit concurrent comparisons. In anticipation of this threat to the protocol we have engaged each of the EMS medical directors in the 
decision to participate. The window of the trial has been confirmed to correspond to the planned changes in the services considering a change.

We have planned a prospective cohort study to compare outcomes across two different prehospital interventions (12-lead and 3-lead) and two system changes (transfer to closest hospital versus bypass closest hospital to transfer directly to a PCI capable hospital) that do not lend themselves to evaluation by a randomized controlled trial. We anticipate there will be challenges related to ethical and privacy, oversight of data guardian abstraction, timeliness of implementation, and technological advances. We hope that this evaluation may be helpful to those involved in developing and enhancing multidisciplinary systems of care including EMS services to advance local care of patients with STEMI and to inform policy decision making and evidence based budgetary decisions that ultimately will affect care across the Province.

\section{Additional material}

Additional file 1: PREDICT Prehospital Variables - Structured data set with variables abstracted from Ambulance Call Reports (ACRs) Additional file 2: PREDICT Hospital Variables - Structured data set with variables abstracted from hospital charts.

\section{List of abbreviations}

ECG: Electrocardiogram; PHECG: Prehospital electrocardiogram; STEMI: ST segment elevated myocardial infarction; EMS: Emergency Medical Services; PCI: Percutaneous coronary intervention; ED: Emergency Department; AMI: Acute myocardial infarction

\section{Acknowledgements}

We would like to acknowledge and thank participating 3 regional Base Hospital programs, their medical directors, management and staff, 12 participating EMS services, all prehospital and inhospital data guardians and members of Rescu team: Eileen O'Connor, Andrew Brooks, Precilla D'Souza and Shane Klein for their contribution to PREDICT.

Ontario Ministry of Health and Long Term Care (MOHLTC) funding has been acquired through an independent research grant awarded to Mr. Ron Goeree through the Programs for Assessment of Technology in Health (PATH) Research Institute. The authors would like to acknowledge the support of the Medical Advisory Secretariat, Ontario Ministry of Health and Long-term Care and the Ontario Health Technology Advisory Committee (OHTAC). VER received a Junior Personnel Award/Health Services/Population Health Post-Doctoral Fellowship from the Heart and Stroke Foundation of Ontario (HSFO). DOR received a Career Scientist Award from MOHLTC.

\section{Author details}

${ }^{1}$ Rescue, Keenan Research Centre, Li Ka Shing Knowledge Institute, St. Michael's Hospital, University of Toronto, Toronto, Ontario, Canada. ²Division of Emergency Medicine; Department of Medicine, University of Toronto, Toronto, Ontario, Canada. ${ }^{3}$ Health Policy, Management \& Evaluation, University of Toronto, Toronto, Ontario, Canada. ${ }^{4}$ Programs for Assessment of Technology in Health (PATH) Research Institute, St., Joseph's Healthcare Hamilton, Hamilton, Ontario, Canada, Department of Clinical Epidemiology and Biostatistics, Faculty of Health Sciences, McMaster University, Hamilton, Ontario, Canada. ${ }^{5}$ Sunnybrook Osler Centre for Prehospital Care, Sunnybrook Health Sciences Centre, Division of Emergency Medicine, Department of Family and Community Medicine, University of Toronto, Toronto Ontario, Canada. ${ }^{6}$ Toronto Emergency Medical Services, Canada.

\section{Authors' contributions}

RG obtained funding for this study. All authors contributed to the study design and the development of the protocol. WR, CZ and RC contributed to the design of PREDICT web based interface. VER will carry out the study under the guidance of LJM. VER drafted the methods manuscript and all authors contributed to the various iterations prior to publication. All authors read and approved the final manuscript.

\section{Competing interests}

The authors declare that they have no competing interests.

Received: 27 November 2010 Accepted: 29 March 2011

Published: 29 March 2011

\section{References}

1. Population Statistics. In Canadian Vital Statistics. Volume 2005. Statistics Canada; 2001.

2. Wilson $E$, Wielgosz $A$ : The changing face of heart disease and stroke in Canada - release of the fifth report from the Canadian Heart and Stroke Surveillance system. Can J Cardiol 1999, 15(10):1075-1079.

3. Jackevicius CA, Alter D, Cox J, Daly P, Goodman S, Filate W, Newman A, Tu JV: Acute treatment of myocardial infarction in Canada 1999-2002. Can J Cardiol 2005, 21(2):145-152.

4. Schull MJ, Vermeulen M, Donovan L, Newman A, Tu JV: Can the wrong statistic be bad for health? Improving the reporting of door-to-needle time performance in acute myocardial infarction. Am Heart J 2005, 150(3):583-587.

5. Schull MJ: What are we waiting for? Understanding, measuring and reducing treatment delays for cardiac patients. Emerg Med Australas 2005, 17(3):191-192.

6. Labinaz M, Swabey $T$, Watson R, Natarajan M, Fucile W, Lubelsky B, Sawadsky B, Cohen E, Glasgow K: Delivery of primary percutaneous coronary intervention for the management of acute ST segment elevation myocardial infarction: summary of the Cardiac Care Network of Ontario Consensus Report. Can J Cardiol 2006, 22(3):243-250.

7. Ryan TJ, Antman EM, Brooks NH, Califf RM, Hillis LD, Hiratzka LF, Rapaport E, Riegel B, Russell RO, Smith EE, et al: 1999 update: ACC/AHA Guidelines for the Management of Patients With Acute Myocardial Infarction: Executive Summary and Recommendations: A report of the American College of Cardiology/American Heart Association Task Force on Practice Guidelines (Committee on Management of Acute Myocardial Infarction). Circulation 1999, 100(9):1016-1030.

8. Feasibility, safety, and efficacy of domiciliary thrombolysis by general practitioners: Grampian region early anistreplase trial. GREAT Group. BMJ 1992, 305(6853):548-553.

9. Prehospital thrombolytic therapy in patients with suspected acute myocardial infarction. The European Myocardial Infarction Project Group. N Engl J Med 1993, 329(6):383-389.

10. Weaver WD, Cerqueira M, Hallstrom AP, Litwin PE, Martin JS, Kudenchuk PJ, Eisenberg M: Prehospital-initiated vs hospital-initiated thrombolytic therapy. The Myocardial Infarction Triage and Intervention Trial. JAMA 1993, 270(10):1211-1216.

11. Morrison LJ, Verbeek PR, McDonald AC, Sawadsky BV, Cook DJ: Mortality and prehospital thrombolysis for acute myocardial infarction: A metaanalysis. JAMA 2000, 283(20):2686-2692.

12. Morrison LJ, Brooks S, Sawadsky B, McDonald A, Verbeek PR: Prehospital 12-lead electrocardiography impact on acute myocardial infarction treatment times and mortality: a systematic review. Acad Emerg Med 2006, 13(1):84-89.

13. 2005 American Heart Association Guidelines for Cardiopulmonary Resuscitation and Emergency Cardiovascular Care. Circulation 2005 112(24 Suppl):IV1-203.

14. 2005 International Consensus on Cardiopulmonary Resuscitation and Emergency Cardiovascular Care Science with Treatment Recommendations. Part 5: Acute coronary syndromes. Resuscitation 2005, 67(2-3):249-269.

15. Curtis JP, Portnay EL, Wang Y, McNamara RL, Herrin J, Bradley EH, Magid DJ, Blaney ME, Canto JG, Krumholz HM: The pre-hospital electrocardiogram and time to reperfusion in patients with acute myocardial infarction, 2000-2002: findings from the National Registry of Myocardial Infarction4. J Am Coll Cardiol 2006, 47(8):1544-1552. 
16. Cantor WJ, Burnstein J, Choi R, Heffernan M, Dzavik V, Lazzam C, Duic M Fitchett D, Tan M, Wawrzyniak J, et al: Transfer for urgent percutaneous coronary intervention early after thrombolysis for ST-elevation myocardial infarction: the TRANSFER-AMI pilot feasibility study. Can J Cardiol 2006, 22(13):1121-1126.

17. de Labriolle A, Pacouret G, Giraudeau B, Fremont B, Desveaux B, Quilliet L, Fauchier $L$, Charbonnier B: Effect of time to treatment and age on one year mortality in acute STEMI: difference between thrombolysis and primary percutaneous coronary intervention. Arch Cardiovasc Dis 2008, 101(1):48-54.

18. Morrison DA, Berman M, El-Amin O, McLaughlin RT, Bates ER: Emergency percutaneous coronary intervention $(\mathrm{PCI})$ for the care of patients with ST-elevation myocardial infarction (STEMI). Minerva Cardioangiol 2007, 55(5):593-623.

19. Terkelsen CJ, Lassen JF, Norgaard BL, Gerdes JC, Poulsen SH, Bendix K Ankersen JP, Gotzsche LB, Romer FK, Nielsen TT, et al: Reduction of treatment delay in patients with ST-elevation myocardial infarction: impact of pre-hospital diagnosis and direct referral to primary percutanous coronary intervention. Eur Heart J 2005, 26(8):770-777.

20. Nallamothu BK, Bates ER, Wang Y, Bradley EH, Krumholz HM: Driving times and distances to hospitals with percutaneous coronary intervention in the United States: implications for prehospital triage of patients with STelevation myocardial infarction. Circulation 2006, 113(9):1189-1195.

21. Bradley EH, Herrin J, Wang Y, Barton BA, Webster TR, Mattera JA, Roumanis SA, Curtis JP, Nallamothu BK, Magid DJ, et al: Strategies for reducing the door-to-balloon time in acute myocardial infarction. $N$ Engl J Med 2006, 355(22):2308-2320.

22. Peterson ED, Ohman EM, Brindis RG, Cohen DJ, Magid DJ: Development of systems of care for ST-elevation myocardial infarction patients: evaluation and outcomes. Circulation 2007, 116(2):e64-67.

23. Chen J, Krumholz HM, Wang Y, Curtis JP, Rathore SS, Ross JS, Normand SL, Schreiner GC, Mulvey G, Nallamothu BK: Differences in patient survival after acute myocardial infarction by hospital capability of performing percutaneous coronary intervention: implications for regionalization. Arch Intern Med 2010, 170(5):433-439.

24. Cardiac Care Network of Ontario: Access to Urgent PCI for ST Segment Elevation Myocardial Infarction. 2004

25. Ontario Health Technology Advisory Committee: OHTAC Recommendation. Primary Angioplasty. 2004, 1-5.

26. Ontario Health Technology Advisory Committee: Primary Angioplasty for the Treatment of Acute ST-Segment Elevated Myocardial Infarction. An Evidence-Based Analysis. Ontario Health Technology Assessment Series 2004, 4:1-65.

27. Statistics Canada: Portrait of the Canadian Population in 2006 Census. Population and Dwelling Counts, 2006 Census. Catalogue no 97-550-XIE 2006.

28. Armstrong PW: A comparison of pharmacologic therapy with/without timely coronary intervention vs. primary percutaneous intervention early after ST-elevation myocardial infarction: the WEST (Which Early STelevation myocardial infarction Therapy) study. Eur Heart J 2006, 27(13):1530-1538.

29. Canto JG, Zalenski RJ, Ornato JP, Rogers WJ, Kiefe Cl, Magid D, Shlipak MG, Frederick PD, Lambrew CG, Littrell KA, et al: Use of emergency medical services in acute myocardial infarction and subsequent quality of care: observations from the National Registry of Myocardial Infarction 2. Circulation 2002, 106(24):3018-3023.

30. Antman EM, Anbe DT, Armstrong PW, Bates ER, Green LA, Hand M, Hochman JS, Krumholz HM, Kushner FG, Lamas GA, et al: ACC/AHA guidelines for the management of patients with ST-elevation myocardial infarction: a report of the American College of Cardiology/American Heart Association Task Force on Practice Guidelines (Committee to Revise the 1999 Guidelines for the Management of Patients with Acute Myocardial Infarction). Circulation 2004, 110(9):e82-292.

31. Armstrong PW, Bogaty P, Buller CE, Dorian P, O'Neill BJ: The 2004 ACC/ AHA Guidelines: a perspective and adaptation for Canada by the Canadian Cardiovascular Society Working Group. Can J Cardiol 2004 20(11):1075-1079.

32. Antman EM, Hand M, Armstrong PW, Bates ER, Green LA, Halasyamani LK, Hochman JS, Krumholz HM, Lamas GA, Mullany CJ, et al: 2007 Focused Update of the ACC/AHA 2004 Guidelines for the Management of Patients With ST-Elevation Myocardial Infarction: a report of the
American College of Cardiology/American Heart Association Task Force on Practice Guidelines: developed in collaboration With the Canadian Cardiovascular Society endorsed by the American Academy of Family Physicians: 2007 Writing Group to Review New Evidence and Update the ACC/AHA 2004 Guidelines for the Management of Patients With STElevation Myocardial Infarction, Writing on Behalf of the 2004 Writing Committee. Circulation 2008, 117(2):296-329.

33. Sex differentials in life expectancy and mortality in developed countries: an analysis by age groups and causes of death from recent and historical data. Popul Bull UN 1988, 25:65-107.

34. Life expectancy. Health Rep 1999, 11(3), 9-24(Eng); 29-24(Fre).

35. Wilkins K: Predictors of death in seniors. Health Rep 2006, 16(Suppl):57-67.

36. Bender R: Impact of new population estimates on health and vital statistics. Health Rep 1995, 7(1):7-18, 17-20.

37. Manuel DG, Schultz SE, Kopec JA: Measuring the health burden of chronic disease and injury using health adjusted life expectancy and the Health Utilities Index. J Epidemiol Community Health 2002, 56(11):843-850.

38. Canadian Institutes of Health Research NSaERCoC, Social Sciences and Humanities Research Council of Canada: Tri-Council Policy Statement: Ethical Conduct for Research Involving Humans. 1998, (with 2000, 2002 and 2005 amendments)

39. Rocher G: Origin and development of the Tri-Council Policy Statement on the Ethics of Research Involving Humans. NCEHR Commun 1999, 9(2):4-6.

40. Reid L, Krahn T: Minimal risk in the Tri-Council Policy Statement. Health Law J 2007, 15:469-513

\section{Pre-publication history}

The pre-publication history for this paper can be accessed here: http://www.biomedcentral.com/1471-227X/11/4/prepub

doi:10.1186/1471-227X-11-4

Cite this article as: Morrison et al:: Prehospital evaluation and economic analysis of different coronary syndrome treatment strategies - PREDICT Rationale, Development and Implementation. BMC Emergency Medicine 2011 11:4.

\section{Submit your next manuscript to BioMed Central and take full advantage of:}

- Convenient online submission

- Thorough peer review

- No space constraints or color figure charges

- Immediate publication on acceptance

- Inclusion in PubMed, CAS, Scopus and Google Scholar

- Research which is freely available for redistribution

Submit your manuscript at www.biomedcentral.com/submit
C Biomed Central 